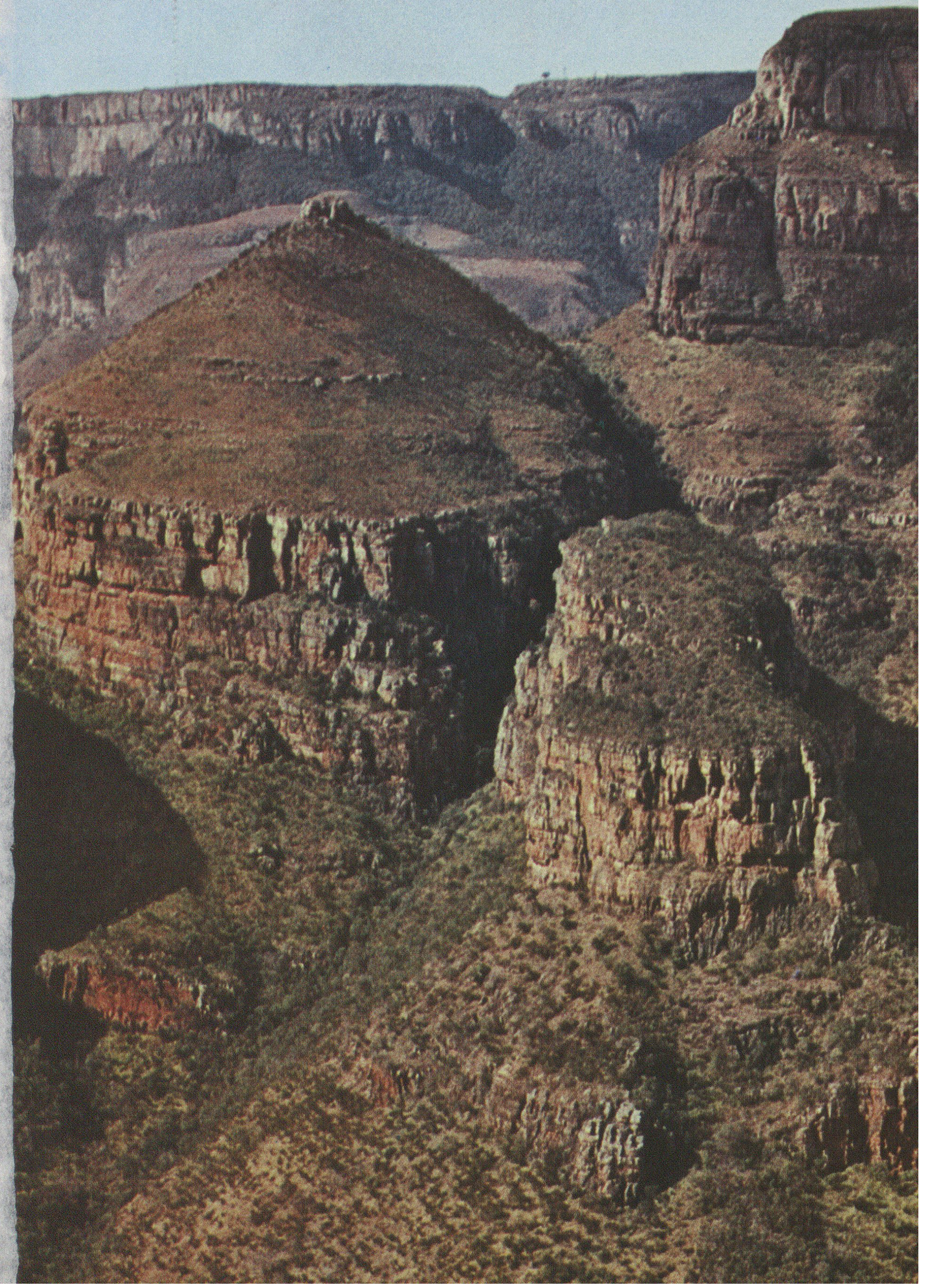




\section{Blyde River und Umgebung (Süd-Afrika)}

Die geologische Geschichte Süd-Afrikas reicht in die frühesten Erdzeitalter zurück. Im Osten der Provinz Transvaal finden wir Gesteinsserien, welche mit einem Alter von mehr als 4000 Millionen Jahren zu den ältesten der Erde gehören. In diesem Teil Süd-Afrikas ist auch die "Grosse Steilstufe" (The Great Escarpment) in den Drakensbergen von Transvaal am eindrücklichsten ausgebildet. "Die Grosse Steilstufe ist vom physischen wie vom wirtschaftlichen Gesichtspunkte aus die bei weitem wichtigste Naturgegebenheit des Subkontinentes" schrieb WELLINGTON (1955, p. 39).

Die "Alten Granite" des Grundgebirges bilden im östlichen Transvaal die Basis der geologischen Strukturen. Im Laufe einer langen geologisch-morphologischen Geschichte wurden sie von sedimentären Deckgebirgen, welche in die drei Perioden Witwatersrand, Transvaal und Karroo gegliedert werden, überlagert. Witwatersrand- und Transvaal-Deckgebirge schei- nen in langgezogenen Sedimentationsbecken, welche sich über den grössten Teil von Transvaal erstrecken, abgelagert worden zu sein. Gegen das Ende der Transvaal-Periode drangen basische und saure Magmen in ungeheuren Massen in die oberen Teile des Deckgebirges ein, die als "Bushveld-Komplex" bezeichnet werden. In der Folge senkten sich die zentralen Teile des Bushveld-Beckens unter dem Gewicht der Intrusionen und die Sedimente der Randzone wurden schräg aufgerichtet; im östlichen Transvaal beobachtet man ein Einfallen von $8-12$ gegen Westen. Eine der drei Unterabteilungen der Transvaalserie (die "Black Reef Group", bestehend aus äusserst widerständigen Quarziten) wurde so in jene Lage gebracht, die 150 Millionen Jahre später für die Bildung der Grossen Steilstufe bestimmend werden sollte.

Während der folgenden Karroo-Periode wurde der ganze Subkontinent mit Sedimenten eingedeckt. Lange nach der Ablagerung der Karroo-

Fig. 1 Das Blyde River-Gebiet (Große Steilstufe) im geologischen Zusammenhang

W

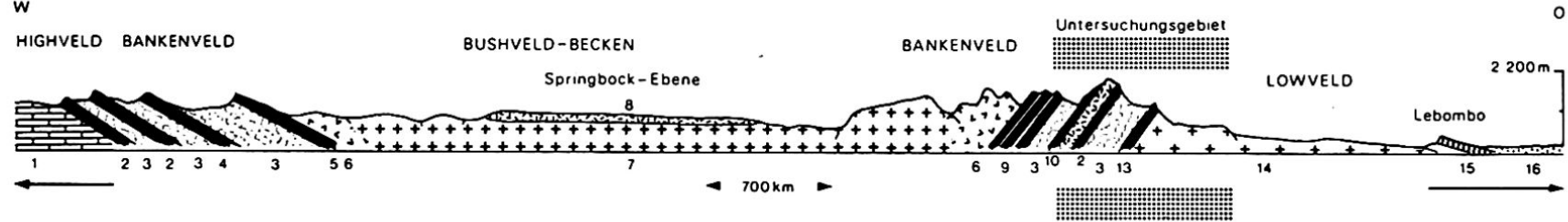

Sedimente der Kreide und des Tertiärs (16)

Karroo-System: $\quad$ Sedimente und Laven (8)

Lebombo Laven (15)

Bushveld-Komplex: Norite (6)

Granite (7)
Transvaal-System: Quartzite: Timeball Hill (2)

Daspoort (4), Magaliesberg (5), Witrand (9), Dewal Heuvel (10) Black Reef (13)

Tonschiefer (3)

Dolomite (1)

Alte Granite (14)

sedimente begann der Gondwanakontinent in einzelne Teile zu zerbrechen. An der Grenze von Moçambique und der Republik Süd-Afrika bildete sich so entlang den Lebombobergen in spättertiärer Zeit erstmals eine Steilstufe; ausserdem drangen an dieser Bruchlinie die Stormberg-Laven der Karroo an die Oberfläche. Von dieser anfänglichen und strukturell bestimmten Lage wurde durch Verwitterung und Abtrag die Steilstufe dauernd gegen Westen zurückverlegt, bis die äusserst resistenten Quarzite des Black Reef zum mindesten

BOOYSEN J. J. , Research-Lecturer, Dept. of Geography, The University of Pretoria, Pretoria 0002 
Fig. 2 Die Periode des Goldbergbaues

Fig. 3 Die Periode der Forstwirtschaft

Fig. 4 Die Periode des Tourismus
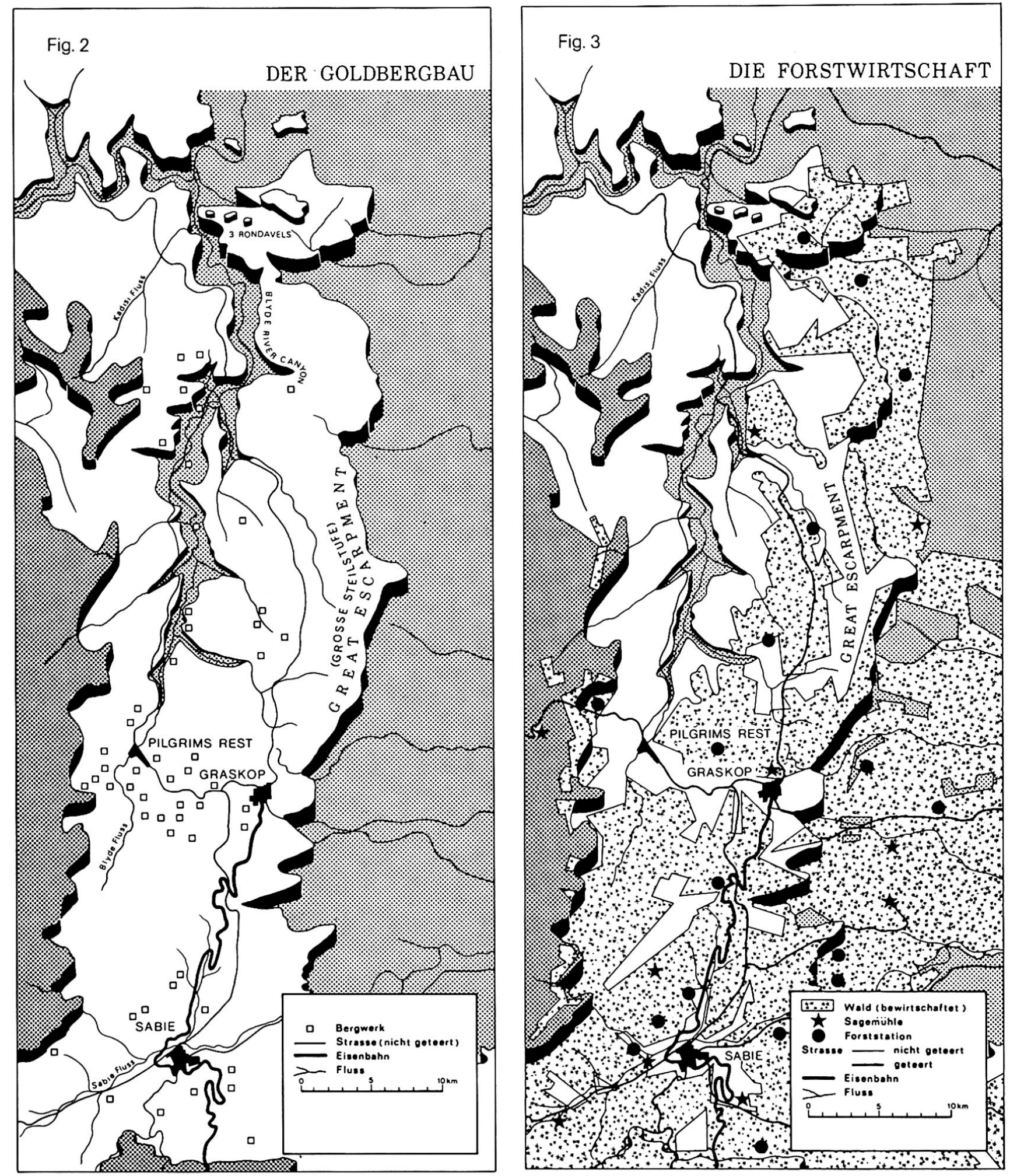


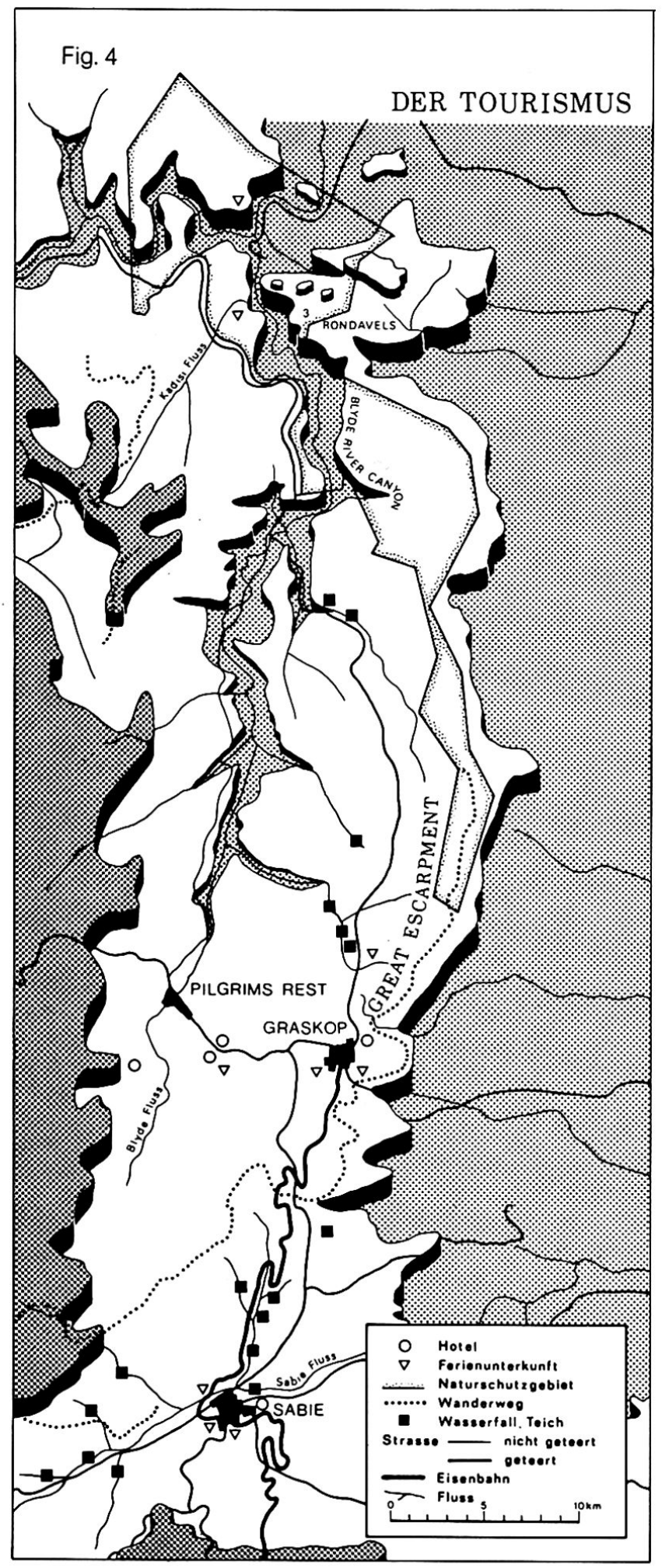

vorübergehend den Vorgang zum Stehen brachten. Das ganze Deckgebirge fiel im Lowveld dieser Rückverwitterung zum Opfer und heute liegen hier die Alten Granite an der Oberfläche.

Jüngere Hebungen leiteten einen neuen morphologischen Zyklus ein. Die Flüsse, welche über die Steilstufe ostwärts zum Lowveld flossen, begannen tiefe Schluchten auszuräumen. Eine davon ist der Blyde River Canyon. Hangverwitterung führte in den schwach nach Westen einfallenden Schichten zu bizarren Formen, von denen das Farbbild die "Drei Rundhütten" (The Three Rondavels) zeigt; diese Bezeichnung wurde wegen ihrer Aehnlichkeit mit den Hütten der schwarzen Bevölkerung gewählt. Neben der landschaftlichen Schönheit sollte später wichtig. werden, dass zur Zeit der Bushveld-Intrusionen Gold in verschiedenartiger Weise abgelagert wurde. Teilweise handelt es sich dabei um Verdrängungslagerstätten (goldführende Quarzla ger in den Dolomiten über dem Black Reef Quarzit), teilweise um senkrechte Quarzgänge oder auch Quarzlinsen - zudem wurde Gold längs Bächen und Flüssen in den Alluvionen abgelagert.

Es war im Jahr 1873, als ein alter Prospektor mit Namen Alec Patterson seinen Weg in die Schluchten des Blyde Rivers fand. Im mittleren Abschnitt des Tales stiess Patterson auf Gold - unmittelbar nachher fand eine eigentliche Prozession in das Fundgebiet stat, denn keine Neuigkeit verbreitet sich so rasch wie die Kunde von einem Goldfund. Gegen Ende des Jahres 1874 zählte man im Umkreis der kleinen Bergwerkssiedlung Pilgrims Rest 1500 Goldsucher und 4000 verschiedene Schürfgebiete (BULPIN, (?), p. 114). Die Orte Pilgrims Rest, Sabie und Graskop blühten rasch auf, Strassen und Luftseilbahnen wurden gebaut, um die schlecht zugänglichen Teile der Goldzone zu erschliessen, und ein Wasserkraftwerk wurde in der grossen Schlucht, durch die der Blyde River seinen Weg zum Lowveld findet, gebaut (CARTWIGHT, 1962, p. 164). Diese blühende Goldzeit ging jedoch rasch zu Ende. Die Goldseifen waren rasch ausgebeutet, die oberflächlichen Primärlagerstätten bald erschöpft; als die tieferliegenden sulfidischen 
Erze erreicht wurden, ergaben sich unüberwindliche Schwierigkeiten bei deren Verarbeitung. Die Goldgewinnung war im Vergleich mit jener am Witwatersrand (Johannesburg) nicht konkurrenzfähig und bald waren nur noch einige wenige kapitalkräftige Gesellschaften in unserem Gebiete tätig. Die höchste Produktion wurde 1910 mit 150'000 Unzen Gold ausgewiesen; 1930 waren es noch 100'000 und 1970 nur noch magere 10'000 Unzen Gold. Nach 1960 war nur noch eine einzige Gesellschaft (Transvaal Gold Mining Estates) tätig und sicherte die Existenz von Pilgrims Rest; 1971 stellte aber auch sie ihren Betrieb ein (NAPPES, 1977, p. 50). Es genügt, FREEMAN (1967, p. 138) zu zitieren, der schrieb: "Extraktive Industrien haben seit Menschengedenken den betreffenden Gebieten nur relativ kurze Prosperität, gefolgt von schliesslichem Zerfall, gebracht". So kehrten erneut Ruhe und Frieden im Tal des Blyde Rivers mit seinen Drei Rondavels ein.

In dem Masse wie die Bedeutung des Goldes abnahm, entwickelte sich gewissermassen als ein Nebenprodukt des Bergbaues eine ausgedehnte Forstwirtschaft. Ihre Anfänge ergaben sich zwangsläufig, weil kurz nach Ausbruch der Goldperiode im Gebiet des Blyde Rivers die vorhandenen Waldbestände zur Gewinnung von Brenn- und Grubenholz geschlagen wurden. Einige Betriebsleiter erkannten früh die Notwendigkeit von Wiederaufforstungen und 1904 waren schon 140 ha hauptsächlich mit Euca lyptus bepflanzt. Man erkannte rasch, dass die Kombination von hohen Niederschlägen, die Nebelzone an der Ostflanke der Drakensberge, das warme Klima und die guten Böden dieses Gebiet zum besten Forstareal von Südafrika machten. Freilich, die Anfänge waren zögernd, doch schon nach dem Ersten Weltkrieg und vor allem in den $F$ ünfzigerjahren wurden in zunehmender Zahl Sägemühlen und holzverarbeitende Industrien errichtet. Tausende von Hektaren wurden mit Pinus patula und Pinus caribaea bepflanzt. 1975 zählte man 385'000 ha Forste, was $20 \%$ der kultivierten Waldfläche der Republik ausmachte. Im gleichen Jahr erreichte die Holzindustrie die höchsten Produktionszahlen. Im Gegensatz zu Gold handelt es sich in diesem Falle nicht um Raubbau, doch sind die wirtschaftlichen Entwicklungs- möglichkeiten eher beschränkt. Im ganzen östlichen Transvaal wurden 1975 nur 57 Weisse und 746 Schwarze in diesem Wirtschaftszweig beschäftigt. Die Forstwirts chaft ist, was den Einfluss auf die wirtschaftliche Entwicklung der Region betrifft, keine Alternative zum Goldbergbau.

Die Landschaft des Blyde River Canyons wurde vom Menschen vorerst mit Bezug auf den Goldbergbau und später, als mögliche Alternative, die Forstwirtschaft gesehen und gewertet. Als vorläufig letzte Nutzungsart reihte sich in diese Sukzession mit rasch zunehmender Bedeutung der Tourismus ein. Das Great Escarpment war von der Natur mit grossartigen Landschaftsszenerien ausgestattet worden. Dank seiner hohen Berge, der üppigen Vegetation, der zahlreichen Wasserfällen, Schluchten und Wasserbecken, aber auch wegen seinen milden Wintern und den historischen Stätten aus der Zeit des Goldbergbaues wie des ersten Burenkrieges entwickelte sich das Blyde River Gebiet zu einem der wichtigsten Touristikzentren von Süd-Afrika. Von 1975 bis 1976 betrug die Zunahme der Touristen $46 \%$. Die 5.5 Millionen Rand (ca. $15 \mathrm{Mil}-$ lionen sFr.) Einkünfte aus dem Tourismus machen schon ungefähr einen Drittel aller Einkünfte der Region aus! 1974 besuchten $112^{\prime} 000$ Touristen das Gebiet von Blyde River und Umgebung bei einer mittleren Aufenthalts dauer von fast 5 Tagen (SWART, 1975, p. 17). Der Tourismus veränderte auch in gewissem Masse das landschaftliche Bild. Der Unterkunftsort "F.H. Odendaal" bietet 720 Touristen Platz; von hier aus geniesst man einen grossartigen Blick auf die Drei Rondavels (SWART, 1975, p. 18). Pilgrims Rest wurde 1973 zum 'national monument' erklärt und als Ganzes restauriert. Nach SWART besuchen die. meisten Touristen von den genannten Standorten aus in Tagesexkursionen Oertlichkeiten von besonderem Interesse und landschaftlicher Schönheit und verwenden hierfür $69 \%$ ihrer Touristikzeit.

So schliesst sich, was man im geographischen Sprachgebrauch als "Perzeptions-Zyklus" bezeichnen kann: Zuerst das Gold, dann die Forstwirtschaft und als Drittes die landschaftliche Schönheit in ihrer Bedeutung für den Tourismus. 


\section{Literaturverzeichnis}

BARNARD, W. S. , SMIT, P. and VAN ZYL, J.A., 1972: Suid-Afrika - die land en sy streke, Nasou Beperk.

BULPIN, T. V., (?): Lost trails in the Low Veld, Hodder and Stoughton.

CARTWRIGHT, A. P. , 1962: Valley of gold, Howard Timmins.

CHRISTOPHER, A. J. , 1973: Environmental perception in Southern Africa, The South African Geographical Journal, 55, 14-22.

HAMILTON, G.N.G. and COOKE, H. B. S. , 1965: Geology for South African students, Central News Agency.

NAPPER, D. G. , 1976: Weekender and holiday guide, Dagmar Publications.

NICOL, I. G. , 1964: A comparitive study of river capture in the Great Escarpment area of the Eastern Transvaal, M. Sc. thesis, Univer- sity of South Africa.

SWART,P.E., 1975: Die invloed van 'n veranderende hulpbronbasis op streeksontwikkeling in die Kruinroetegebied, Hons. project, University of Pretoria.

TALJAARD, M. S. , 1948: Oor berge en vlaktes, Universiteituitgewers.

VAN EEDEN, O.R., 1972: Geology of the Republic of South Africa, Government Printer.

WYBERGH, W. J., 1925: Economic geology of Sabie and Pilgrims Rest, Geological Survey, memoir no 23, Government Printer.

Das Farbbild wurde freundlicherweise vom Verlag Kümmerly + Frey zur Verfügung gestellt. Es stammt aus dem Bildband "Südafrika-Land ohne Beispiel" von Hans Jenny, K \& F, Bern, 1976.

\section{Literaturbesprechungen}

OOI Jin Bee: Pensinsular Malaysia. Longman, London and New York, xvi, +437 , Fig. , £7. 95, 1976 .

Professor Ooi ist Direktor des Geographischen Institutes der Universität in Singapore. 1963 veröffentlichte er unter dem Titel "Land, people, and economy in Malaya" den Vorläufer zu dem hier besprochenen Buch, welches in Longman's "geographies for advanced study" aufgenommen wurde. Ooi ist der kompetente Kenner der Materie, die er wie in der früheren Auflage in die drei Hauptkapitel "land, people, economy"gliedert. Auch die weitere Gliederung ist ausgesprochen traditionell, wobei offen bleiben soll, ob sich darin die Absicht des Herausgebers oder der Autor wiederspiegelt. Dies ist heute, wo man immer von problem-orientierter Geographie spricht, nicht unbedingt selbstverständlich. Natürlich werden die Materialien dafür sauber geordnet bereitgestellt, doch bleibt es weitgehend dem Leser überlassen, wie er sie im angedeuteten Sinne verwendet. Wer die Probleme Malayas kennt, findet in diesem Buch eine Fülle von vertrauenswürdigem Material zu deren Beurteilung - wer damit nicht vertraut ist, wird manchmal Mühe haben, die Probleme zu erkennen. Eines ist sicher:Peninsular Malaysia dürfte heute das beste Werk sein zur objektiven Information. In dieser Richtung bleibt es auch keineswegs an der Oberfläche, sondern schürft tief. Wertvoll ist für Viele ein Glossar malayischer Ausdrücke. Auf der anderen Seite ist es zweifellos verdienstlich, ausführliche Literaturverzeichnise in einem Lehrbuch aufzunehmen - man frägt sich nur, ob es sinnvoll ist, wenn ein grosser Teil dieser Literatur (beispielsweise Regierungspublikationen) aus serhalb Malaysias kaum vorhanden sein dürfte. GH 2/1977
Hans Boesch 